\title{
Detection of Villous Atrophy Using Endoscopic Images for the Diagnosis of Celiac Disease
}

\author{
Edward J. Ciaccio • Suzanne K. Lewis • \\ Peter H. Green
}

Published online: 24 March 2013

(C) Springer Science+Business Media New York 2013

\section{Background}

The most common cause of villous atrophy is celiac disease. The current "gold standard" in the diagnosis of celiac disease is the demonstration of villous atrophy in duodenal biopsies, which are usually obtained during a fiber-optic endoscopic foregut examination [1]. Since conventional upper tract endoscopy does not extend past the duodenum, it is not suitable to determine the length of pathological involvement of the small intestine. Moreover, since duodenal villous atrophy is often patchy [2,3], gross mucosal features, such as scalloping of duodenal folds, reduction of duodenal folds, mosaic pattern (cobblestone appearance of the mucosal surface), and mucosal fissuring [4-7], can be used to identify involved areas. It is nevertheless possible to miss the involved areas since the gross manifestations of villous atrophy may be absent in mild disease $[8,9]$.

\section{Enhancement of Endoscopic Images by i-Scan and Other Means}

A number of recently developed techniques have been found useful to enhance the endoscopic images of villous atrophy, including magnification endoscopy [10], with or without chromoendoscopy [11], immersion techniques [12], and confocal endoscopy [13-16]. However, some of these methods typically require sophisticated instrumentation, special training, and expertise. One recent technology

\section{E. J. Ciaccio $(\square)$ · S. K. Lewis · P. H. Green}

Department of Medicine, The Celiac Disease Center, Columbia

University Medical Center, Harkness Pavilion 934, 180 Fort

Washington Avenue, New York, NY 10032, USA

e-mail: ciaccio@columbia.edu described in this issue of Digestive Diseases and Sciences termed i-Scan (Pentax, Tokyo, Japan), is a commercially available, software-based, virtual chromoendoscopy method [17] that can be used in real time without the need for extensive training. The technique improves tissue contrast by spectral estimation of the digitized images and therefore does not require instrumentation accessories such as optical filters within the video endoscope [18]. For image enhancement, the i-Scan technology has the following three software-derived modalities [19]:

1. Surface enhancement-recognition of feature edges is achieved based on differences in luminance intensity. Compared with normal images, surface-enhanced images have the same brightness and only slight color changes.

2. Contrast enhancement-differences in image structure are accentuated by adjusting areas of lower luminance intensity upward in the blue color component and downward in the red and green color component. This provides improved contrast of the mucosal surface as well as capillary patterns [18]. Compared with normal images, contrast-enhanced images also have the same brightness and only slight changes to color.

3. Tone enhancement-the regions of esophagus, stomach, and colon are separately enhanced by modifying the combination of color components contributing to form the color at each pixel, which is done to specifically reflect the quantitative image properties of the individual organ.

The surface and contrast enhancement modalities each consist of three levels (low, medium, and high); for the tone enhancement modality, organ type is selected (esophagus, stomach, or colon) [19]. The three modalities are arranged sequentially, so that more than one modality 
can be applied simultaneously. Switching modalities and enhancement levels is done in real time using a button on the endoscope $[17,20]$. The i-Scan technology is useful to evaluate the gross anatomical structure of the small intestinal mucosal surface, including shape and contour [17]. Therefore, the aforementioned macroscopic features characteristic of villous atrophy can be better detected by i-Scan during endoscopic mucosal examination. Chromoendoscopy combined with high-definition colonoscopy and i-Scan were able to identify lesions that were missed by high-definition endoscopy alone, with i-Scan and chromoendoscopy having equal accuracy for the detection of small neoplasia [18, 21]. Therefore, i-Scan can potentially replace time-consuming chromoendoscopy [18, 21]. To date, no complications have been reported with the use of i-Scan technology [19].

\section{A New Study Involving i-Scan Technology}

In this issue of Digestive Diseases and Sciences, Cammarota et al. [22] report a prospective, single-center study on the diagnostic accuracy of i-Scan technology for the evaluation of the duodenal villous atrophy in patients undergoing upper endoscopy for iron deficiency anemia, diarrhea, abdominal pain, positive anti-endomysial or antitransglutaminase antibodies, and unexplained weight loss, as compared with a "gold standard" of histologic analysis. Two expert endoscopists, blinded to each other's findings and the subject's laboratory and clinical data, evaluated the endoscopic images obtained from the population of 150 subjects. The duodenal villous patterns obtained with i-Scan were classified by the endoscopists as either marked villous atrophy, partial villous atrophy, or normal, and were compared to simultaneously obtained histology, read by blinded pathologists.

The accuracy of i-Scan for detecting marked villous atrophy patterns was $100 \%$, whereas the accuracy for i-Scan detection of partial villous atrophy or normal villous patterns was each $90 \%$. Thus, i-Scan technology was found to be useful to enhance the endoscopic images of the duodenal mucosa for predicting the presence of villous atrophy. Since i-Scan enhances the images in software without the need for dyes and without additional instrumentation, complexity and analysis time were reduced. The excellent classification results suggest that the new technology will be useful for detecting villous atrophy.

Although i-Scan has been shown to be a promising technology, there are limitations to its use. For example, when i-Scan was utilized to characterize small colonic polyps for prediction of histology, it was found that accuracy varied substantially between endoscopists [23]. This suggests that user bias may be an important factor in the efficacy of the technology. Furthermore, for cancer surveillance, it has been recommended that primary screening should be performed with conventional white light endoscopy, after which i-Scan or other digital chromoendoscopy techniques can be used to analyze suspicious lesions [24]. Therefore, i-Scan may be most useful as an adjunctive tool, not as a primary method, for evaluation of small intestinal features.

\section{Future Directions for Endoscopic Image Enhancement}

One possible technique to further determine the degree of villous atrophy is to analyze endoscopic images obtained directly or via wireless capsule using computerized image analysis, which could encompass the entire small intestine, enabling the construction of a spatial map from proximal to distal small intestine to show areas and degrees of villous atrophy [25-27]. This would be useful to determine, for example, efficacy of a gluten-free diet treatment, or to screen patients with suspected villous atrophy who have little or no evident changes that can be detected by conventional endoscopic examination. Quantitative methods can also be used to analyze biopsies [28]. Until the present time, however, the accuracy of all of these quantitative methods depends in part upon limited image resolution, the random and often oblique camera angle with respect to the small intestinal mucosal wall, and in the case of video endoscopic images, the presence of extraneous substances such as air bubbles and opaque fluids within the lumen. Therefore, improved imaging methods would be highly desirable for better quantification and for increased visualization [29].

According to Cammarota et al., i-Scan technology was less accurate in identifying mild-to-moderate villous atrophy. Further refinement of image enhancement or computerized quantification techniques should improve identification of images with lesser degrees of villous atrophy. It is only then that image enhancement techniques could possibly replace direct biopsy $[30,31]$ in the diagnosis of celiac disease.

In addition to studies with larger patient populations, comparison with other techniques, and use of decision analysis to determine costs associated with implementation of the more expensive systems should be performed in future studies. Further investigation using i-Scan would also be used to determine whether celiac disease and other digestive disease can be distinguished from the general population of patients undergoing upper endoscopy for unrelated indications. Moreover, the development of a numerical score associated with i-Scan technology would provide a quantitative means for comparison.

In summary, detection of villous atrophy from endoscopic images can be difficult due to the limited spatial 
resolution and marginally effective enhancement techniques that are available. The authors of "Image-enhanced endoscopy with i-Scan technology for the evaluation of duodenal villous patterns," appearing in this issue of Digestive Diseases and Sciences, present the results of a newly developed i-Scan technique. This novel methodology is promising for improved visualization of endoscopic images when villous atrophy is present in the small intestinal mucosa. Other more quantitative methods may be useful to incorporate with the new i-Scan technology to automatically detect and localize regions of villous atrophy in celiac disease patients.

\section{References}

1. Green PH, Cellier C. Celiac disease. $N$ Engl J Med. 2007;357: 1731-1743.

2. Bonamico M, Mariani P, Thanasi E, et al. Patchy villous atrophy of the duodenum in childhood celiac disease. J Pediatr Gastroenterol Nutr. 2004;38:204-207.

3. Ravelli A, Villanacci V, Monfredini C, Martinazzi S, Grassi V, Manenti S. How patchy is patchy villous atrophy?: Distribution pattern of histological lesions in the duodenum of children with celiac disease. Am J Gastroenterol. 2010;105:2103-2110.

4. Jabbari M, Wild G, Goresky CA, et al. Scalloped valvulae conniventes: an endoscopic marker of celiac sprue. Gastroenterology. 1988;95:1518-1522.

5. Brocchi E, Corazza GR, Caletti G, Treggiari EA, Barbara L, Gasbarrini G. Endoscopic demonstration of loss of duodenal folds in the diagnosis of celiac disease. $N$ Engl J Med. 1988;319:741-744.

6. Maurino E, Capizzano H, Niveloni S, et al. Value of endoscopic markers in celiac disease. Dig Dis Sci. 1993;38:2028-2033.

7. Chang MS, Rubin M, Lewis SK, Green PH. Diagnosing celiac disease by video capsule endoscopy (VCE) when esophogastroduodenoscopy (EGD) and biopsy is unable to provide a diagnosis: a case series. BMC Gastroenterol. 2012;12:90.

8. Dickey W, Hughes D. Prevalence of celiac disease and its endoscopic markers among patients having routine upper gastrointestinal endoscopy. Am J Gastroenterol. 1999;94:2182-2186.

9. Dickey W, Hughes D. Disappointing sensitivity of endoscopic markers for villous atrophy in a high-risk population: implications for celiac disease diagnosis during routine endoscopy. Am J Gastroenterol. 2001;96:2126-2128.

10. Banerjee R, Shekharan A, Ramji C, et al. Role of magnification endoscopy in the diagnosis and evaluation of suspected celiac disease: correlation with histology. Indian $J$ Gastroenterol. 2007;26:67-69.

11. Siegel LM, Stevens PD, Lightdale CJ, et al. Combined magnification endoscopy with chromoendoscopy in the evaluation of patients with suspected malabsorption. Gastrointest Endosc. 1997;46:226-230.

12. Cammarota G, Cesaro P, La Mura R, et al. Role of the "Immersion technique" In diagnosing celiac disease with villous atrophy limited to the duodenal bulb. J Clin Gastroenterol. 2007;41:571-575.

13. Cammarota G, Martino A, Pirozzi GA, et al. Direct visualization of intestinal villi by high-resolution magnifying upper endoscopy: a validation study. Gastrointest Endosc. 2004;60:732-738.
14. Leong RW, Nguyen NQ, Meredith CG et al. In vivo confocal endomicroscopy in the diagnosis and evaluation of celiac disease. Gastroenterology 2008;135:1870-1876

15. Cammarota G, Fedeli P, Gasbarrini A. Emerging technologies in upper gastrointestinal endoscopy and celiac disease. Nat Clin Pract Gastroenterol Hepatol. 2009;6:47-56.

16. Cammarota G, Cesaro P, Cazzato A, et al. The water immersion technique is easy to learn for routine use during EGD for duodenal villous evaluation: a single-center 2-year experience. J Clin Gastroenterol. 2009;43:244-248.

17. Mönkemüller K, Neumann H, Fry LC. Enteroscopy: advances in diagnostic imaging. Best Pract Res Clin Gastroenterol. 2012; 26:221-233.

18. Neumann H, Neurath MF, Mudter J. New endoscopic approaches in IBD. World J Gastroenterol. 2011;17:63-68.

19. Galloro G, Magno L, Ruggiero S, Fusco F, Rappa T. Contribution of new technologies to endoscopic imaging. In: Trecca A, ed. Ileoscopy: technique, diagnosis, and clinical applications. Milan: Springer; 2012, Chapter 4, pp 21-29.

20. Kodashima S, Fujishiro M. Novel image-enhanced endoscopy with i-Scan technology. World J Gastroenterol. 2010;16:10431049.

21. Hoffman A, Kagel C, Goetz M, et al. Recognition and characterization of small colonic neoplasia with high-definition colonoscopy using i-Scan is as precise as chromoendoscopy. Dig Liver Dis. 2010;42:45-50.

22. Cammarota G, Ianiro G, Sparano L, La Mura R, Ricci R, Larocca LM et al. Image-enhanced endoscopy with i-Scan technology for the evaluation of duodenal villous patterns. Dig Dis Sci. 2012. doi:10.1007/s10620-012-2467-y

23. Chan JL, Lin L, Feiler M, Wolf AI, Cardona DM, Gellad ZF. Comparative effectiveness of i-SCAN ${ }^{\mathrm{TM}}$ and high-definition white light characterizing small colonic polyps. World $J$ Gastroenterol. 2012;18:5905-5911.

24. Pittayanon R, Rerknimitr R. Role of digital chromoendoscopy and confocal laser endomicroscopy for gastric intestinal metaplasia and cancer surveillance. World $J$ Gastrointest Endosc. 2012;4:472-478.

25. Ciaccio EJ, Bhagat G, Tennyson CA, Lewis SK, Hernandez L, Green PH. Quantitative assessment of endoscopic images for degree of villous atrophy in celiac disease. Dig Dis Sci. 2011;56:805-811.

26. Ciaccio EJ, Tennyson CA, Lewis SK, Krishnareddy S, Bhagat G, Green PH. Distinguishing patients with celiac disease by quantitative analysis of videocapsule endoscopy images. Comput Methods Prog Biomed. 2010;100:39-48.

27. Ciaccio EJ, Tennyson CA, Bhagat G, Lewis SK, Green PH. Quantitative estimates of motility from videocapsule endoscopy are useful to discern celiac patients from controls. Dig Dis Sci. 2012;57:2936-2943.

28. Ciaccio EJ, Bhagat G, Naiyer AJ, Hernandez L, Green PH. Quantitative assessment of the degree of villous atrophy in patients with coeliac disease. J Clin Pathol. 2008;61:1089-1093.

29. Ciaccio EJ, Tennyson CA, Bhagat G, Lewis SK, Green PH. Transformation of videocapsule images to detect small bowel mucosal differences in celiac versus control patients. Comput Methods Prog Biomed. 2012;108:28-37.

30. Rostom A, Murray JA, Kagnoff MF. American Gastroenterological Association (AGA) institute technical review on the diagnosis and management of celiac disease. Gastroenterology. 2006;131:1981-2002.

31. Gonzalez S, Gupta A, Cheng J, et al. Prospective study of the role of duodenal bulb biopsies in the diagnosis of celiac disease. Gastrointest Endosc. 2010;72:758-765. 